\title{
Experimental study of tensile strength of pharmaceutical tablets: effect of the diluent nature and compression pressure
}

\author{
Audrey Juban ${ }^{1, \star}$, Stéphanie Briançon ${ }^{1, \star \star}$, François Puel ${ }^{2, \star \star \star}$, Thierry Hoc ${ }^{3, \star \star \star \star}$, and Cécile Nouguier-Lehon ${ }^{3, \dagger}$ \\ ${ }^{1}$ Univ Lyon, Universite Lyon 1, CNRS, UMR5007, LAGEP, 43 bd du 11 Novembre 1918, F-69622 LYON, France \\ ${ }^{2}$ LGPM, CentraleSupélec, Université Paris-Saclay, Grande Voie des Vignes, 92295 Châtenay-Malabry, France \\ ${ }^{3}$ Univ Lyon, Ecole Centrale de Lyon, LTDS UMR CNRS 5513, 36 avenue Guy de Collongue, F-69134 ECULLY cedex, France
}

\begin{abstract}
In the pharmaceutical field, tablets are the most common dosage form for oral administration in the world. Among different manufacturing processes, direct compression is widely used because of its economics interest and it is a process which avoids the steps of wet granulation and drying processes. Tablets are composed of at least two ingredients: an active pharmaceutical ingredient (API) which is mixed with a diluent. The nature of the powders and the processing conditions are crucial for the properties of the blend and, consequently, strongly influence the mechanical characteristics of tablets. Moreover, tablets have to present a suitable mechanical strength to avoid crumbling or breaking when handling, while ensuring an appropriate disintegration after administration. Accordingly, this mechanical property is an essential parameter to consider. Experimental results showed that proportion of the diluent, fragmentary (DCPA) or plastic (MCC), had a large influence on the tensile strength evolution with API content as well as the compression load applied during tableting process. From these results a model was developed in order to predict the tensile strength of binary tablets by knowing the compression pressure. The validity of this model was demonstrated for the two studied systems and a comparison was made with two existing models.
\end{abstract}

\section{Introduction}

Pharmaceutical tablet is the most common dosage form for oral administration, mainly because it is an easier way for drug intake by patients. Other reasons for this success are the ease of large-scale production, their safety and easy handling and good stability. Among different manufacturing processes, direct compression is widely used because of its economics interest and its process which avoids the steps of wet granulation and drying processes [1-4]. Tablets are composed of at least two ingredients: an active pharmaceutical ingredient (API) which is mixed with a diluent [5]. Other ingredients could also be included, like lubricants, disintegrating agents, binders, etc. It is well known that the nature of the powders used and the processing conditions are crucial for the properties of the blend (fluidity and compressibility) and, consequently, strongly influence the mechanical characteristics of the obtained tablets $[6,7]$. Tablets have to present a suitable mechanical strength to avoid crumbling or breaking when handling, while ensuring an appropriate disintegration after administration. Accordingly, the tablet tensile strength is an essential parameter to consider [8-11] and is therefore standardly tested during the manufacturing process

\footnotetext{
^e-mail: audrey.juban.pro@gmail.com

$\star \star$ e-mail: stephanie.briancon@univ-lyon1.fr

$\star \star \star$ e-mail: francois.puel@centralesupelec.fr

$\star \star \star \star$ e-mail: thierry.hoc@ec-lyon.fr

†e-mail: cecile.nouguier@ec-lyon.fr
}

[12]. This mechanical characteristic is calculated from the breaking force of the tablet obtained from a standard method, generally using a diametral compression test [13].

The objective of this work is first to experimentally study the effect of operating conditions: composition and compression load (formulation and process parameter, respectively). In a second step, a model allowing the prediction of the tensile strength of binary tablets from the pressure applied during direct tableting process was proposed.

\section{Materials and methods}

\subsection{Powders}

The active pharmaceutical ingredient (API) used in this work is the anhydrous caffeine form I (CFI) obtained by using the same method as [14] from commercial anhydrous caffeine Form II (Cooper). To study the effect of the diluent nature, two different powders were used: microcrystalline cellulose (MCC) Avicel@PH 102 (FMC Health and nutrition) and anhydrous dicalcium phosphate (DCPA) from JRS Pharma. CFI and MCC are known to present a plastic behavior under compression, whereas DCPA exhibits a fragmentary behavior $[5,15,16]$. 


\subsection{Tablet preparation}

Cylindrical tablets were prepared from binary mixtures of CFI (one day after its manufacture) and MCC or DCPA as diluent. Each diluent was mixed in various proportions with caffeine: $10,20,30,40,50,60,78$ and 90 $\mathrm{wt} \%$ of CFI for MCC and 20, 40, 60 and $80 \mathrm{wt} \%$ of CFI for DCPA. The blending was performed with a tridimensional mixer (Turbula $囚 \mathrm{~T} 2 \mathrm{~F}$ ) at a rotational rate of 49 rpm for a duration of 10 minutes. Series of pure CFI and MCC tablets were also made. All tablets were produced at a constant mass (about $300 \mathrm{mg}$ ) by using a compaction simulator Styl'One Classic (MEDEL'PHARM, Beynost, France) and its data acquisition software (Analis, 2.03 version, MEDEL'PHARM). Standard Euro D tools with flatfaces of diameter $11.28 \mathrm{~mm}$ were fitted on the simulator. Refer to [14] for a complete description of this single station press. Tablets were made without lubricant using one main compression, driven by force. To study the effect of compression pressure, tablets were produced under three different compression loads corresponding to 50,100 and $200 \mathrm{MPa}$ in compression pressure, noted $P$ thereafter. According to the European Pharmacopoeia [12], the tablet mass $m$, thickness $t$ and diameter $D$ were measured just after compression, allowing the determination of tablet density and porosity.

\subsection{Diametral compression test}

Diametral compression tests were performed on tablets one day after their fabrication using a tensile test device (ElectroPulsTM E10000, Instron $\AA$, Elancourt, France) and its data acquisition software (WaveMatrixTM, Instron( $)$ ). A sensor of $1 \mathrm{kN}$ was used. Diametral compression tests were performed at constant velocity of 0.1 mm. $\mathrm{min}^{-1}$ with an acquisition rate of $10 \mathrm{~Hz}$. A minimum of three tablets per composition and per compression load were tested. The maximal diametral crushing force $F$ was measured for each tablet and the tensile strength was calculated using the classical equation [17]:

$$
\sigma_{T}=\frac{2 F}{\pi D t}
$$

\section{Results}

\subsection{Tablet tensile strength}

Table 1 reports the tablet tensile strength for all compositions studied. As expected, and in accordance with the literature $[15,18]$, for a given composition, the tablet tensile strength increased with the compression pressure. Moreover, the tensile strengths of tablets containing DCPA were lower than those obtained for CFI/MCC mixtures. For $\mathrm{CFI} / \mathrm{MCC}$ mixtures, tablet tensile strength generally increased with a decreasing of the caffeine content in the formulation. By contrast, with the binary mixtures containing DCPA, the tablet tensile strength slightly increased with the caffeine content. At the lowest compaction pressure $(50 \mathrm{MPa})$ the trend of tablet tensile strength with caffeine
Table 1. Tablet tensile strength ( $\sigma_{T}$ in MPa) obtained from diametral compression test. Average calculated from 3 tablets.

\begin{tabular}{ccccc}
\hline $\begin{array}{c}\text { Diluent } \\
(-)\end{array}$ & $\begin{array}{c}\text { Composition } \\
\text { (wt\% CFI) }\end{array}$ & \multicolumn{3}{c}{$\begin{array}{c}\text { Compression pressure P } \\
\text { (MPa) }\end{array}$} \\
\hline & 100 & $1.08 \pm 0.11$ & $1.84 \pm 0.09$ & $2.39 \pm 0.29$ \\
\hline & 90 & $1.34 \pm 0.05$ & $2.36 \pm 0.09$ & $3.20 \pm 0.10$ \\
& 78 & $1.21 \pm 0.03$ & $2.56 \pm 0.02$ & $4.16 \pm 0.11$ \\
& 60 & $1.65 \pm 0.04$ & $3.34 \pm 0.12$ & $5.41 \pm 0.29$ \\
& 50 & $1.54 \pm 0.02$ & $3.34 \pm 0.06$ & $6.06 \pm 0.17$ \\
$\mathrm{MCC}^{\mathrm{a}}$ & 40 & $1.65 \pm 0.01$ & $3.79 \pm 0.04$ & $6.79 \pm 0.09$ \\
& 30 & $1.50 \pm 0.01$ & $3.60 \pm 0.06$ & $6.48 \pm 0.07$ \\
& 20 & $1.80 \pm 0.01$ & $4.27 \pm 0.06$ & $7.87 \pm 0.12$ \\
& 10 & $1.69 \pm 0.06$ & $4.21 \pm 0.08$ & $8.16 \pm 0.12$ \\
& 0 & $2.00 \pm 0.07$ & $5.04 \pm 0.11$ & $9.73 \pm 0.24$ \\
\hline & 80 & $0.97 \pm 0.04$ & $1.69 \pm 0.05$ & $2.79 \pm 0.09$ \\
DCPA & 60 & $0.74 \pm 0.01$ & $1.33 \pm 0.07$ & $2.52 \pm 0.06$ \\
& 40 & $0.49 \pm 0.03$ & $1.08 \pm 0.05$ & $1.99 \pm 0.06$ \\
& 20 & $0.30 \pm 0.00$ & $0.67 \pm 0.02$ & $1.62 \pm 0.03$ \\
\hline a From [14] & & & &
\end{tabular}

content was more erratic than for the greater compression pressure. This variability can be explained by the tablet porosity dispersion at low pressure.

\subsection{Modeling the tablet tensile strength}

As observed in table 1, the compression pressure, $P$, is the parameter having the greatest impact on the value of the tablet tensile strength, $\sigma_{T}$. Hence, the objective of this section is to propose a model allowing the prediction of the tensile strength of binary tablet by knowing the compression pressure applied during the direct tableting process. Moreover, since the pharmaceutical tablets are usually composed of a minimum of two components, it seems relevant to only consider the two extreme compositions of each studied mixture for the construction of the model. Consequently, a linear regression was plotted for the two extreme formulations of each studied mixture (10 and $90 \mathrm{wt} \%$ of CFI and 20 and $80 \mathrm{wt} \%$ of CFI for MCC and DCPA, respectively). The coefficient of determination $R^{2}$ was always greater than 0.94 . Values of regression parameters obviously depend on each blend and hence on the percentage of CFI. Assuming a linear relationship for these parameters with the caffeine content, the tablet tensile strength can finally be predicted by:

$$
\sigma_{T}=\left(A_{1} \times \mathrm{wt} \% \mathrm{CFI}+A_{2}\right) \times P+\left(B_{1} \times \mathrm{wt} \% \mathrm{CFI}+B_{2}\right)
$$

The values of coefficients $A_{1}, A_{2}, B_{1}$ and $B_{2}$ obtained for the two studied mixtures are given in table 2 .

Table 2. Coefficients required for the application of the proposed model (equation 2)

\begin{tabular}{ccccc}
\hline & $\begin{array}{c}A_{1} \\
(-)\end{array}$ & $\begin{array}{c}A_{2} \\
(-)\end{array}$ & $\begin{array}{c}B_{1} \\
(\mathrm{MPa})\end{array}$ & $\begin{array}{c}B_{2} \\
(\mathrm{MPa})\end{array}$ \\
\hline CFI/MCC & $-3.9 \cdot 10^{-4}$ & 0.0464 & 0.0151 & -0.4356 \\
CFI/DCPA & $5.2 \cdot 10^{-5}$ & 0.0079 & 0.0099 & -0.3733 \\
\hline
\end{tabular}




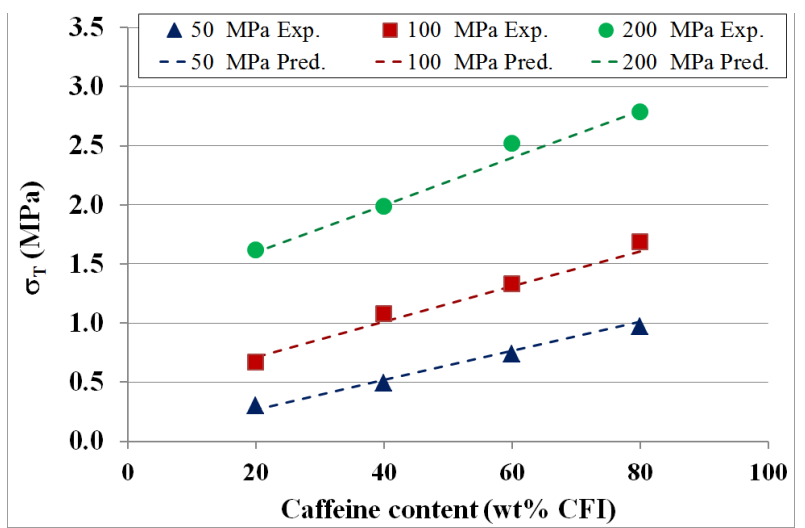

Figure 1. Evolution of experimental tensile strength $\sigma_{T}$ obtained by diametral compression test (filled symbols) and predicted values (lines) versus the percentage of caffeine in tablets for binary mixture of CFI/DCPA

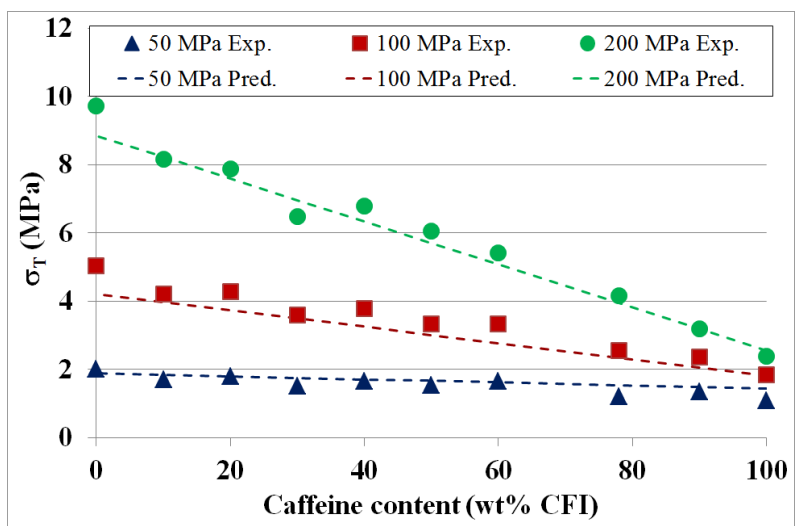

Figure 2. Evolution of experimental tensile strength $\sigma_{T}$ obtained by diametral compression test (filled symbols) and predicted values (lines) versus the percentage of caffeine in tablets for binary mixture of CFI/MCC

\section{Discussion}

\subsection{Validation of the proposed model}

Figures 1 and 2 show the evolution of tablet tensile strength according to the percentage of CFI for binary mixtures containing DCPA and MCC, respectively. The comparison of experimental data (filled symbols) with the values predicted by equation 2 (lines) showed that the proposed model is relevant, and one can note that most of the experimental values were slightly underestimated by the model. Mean relative deviations between predicted and experimental values of tablet tensile strength are of $4.2 \%$ and $8.2 \%$ for binary mixtures of CFI/DCPA and $\mathrm{CFI} / \mathrm{MCC}$, respectively. In the case of CFI/MCC mixture, pure tablets were manufactured and the mean relative deviation was $9.1 \%$ using the data for 0 and 100 wt $\%$, close to value obtained with 10 and $90 \mathrm{wt} \%$. It is worth noting that the proposed model is efficient for binary mixtures composed of two components exhibiting plastic deformation under compression (CFI/MCC) but also for
Table 3. Mean relative deviations between experimental tablet tensile strength and values predicted by models

\begin{tabular}{cccc}
\hline Diluent & Equation (2) & Model [14] & Model [22] \\
\hline MCC & $8.2 \%$ & $12.0 \%$ & $14.6 \%$ \\
DCPA & $4.2 \%$ & $7.8 \%$ & - \\
\hline
\end{tabular}

mixtures containing a plastic and a fragmentary compound (CFI/DCPA).

\subsection{Comparison with models from literature}

Several authors have been interested in the study of tablet tensile strength, looking especially for a method allowing its prediction. Most of them are based on the knowledge of the relative density (or the porosity) of mixture. One can cite the studies based on the percolation theory [18-21] (which also requires several unknown parameters such as critical relative density) and the studies based on the Ryskewitch-Duckworth equation [22, 23]. However, recently a model between the tablet tensile strength and the surface hardness of tablet was also proposed [14]. For more details about these models refer to [14].

In order to test the efficiency of the model proposed in this study, we compared results obtained by equation 2 with values predicted by Wu et al.'s model [22] and Juban et al.'s model [14]. Data required to apply these models were determined using the methodology already described in [14]. Especially, for each composition studied, the relative density and the porosity of tablets were calculated and the hardness measurements were performed by using the same device and method. The mean relative deviations between experimental and predicted data calculated from the three predictive models were given in table 3, showing the good ability for all models to predict the tensile strength of binary tablets. Nevertheless, $\mathrm{Wu}$ et al.'s model could not be applied for binary mixtures composed of CFI/DCPA, because it has been impossible to manufacture pure tablets of DCPA due to important binding and sticking phenomena occurring during the direct tableting process. This problem highlighted a restriction of this model, since some pharmaceutical powders, especially API, do not have the required compression properties such as fluidity and compressibility to form pure tablets $[24,25]$. Moreover, the predicted data calculated from equation 2 and from the model developed in [14] were slightly underestimated compared to the experimental ones, for the two mixtures studied. By contrast, for tablets containing MCC as diluent, the tensile strengths predicted from [22] presented an overestimation.

The main difference between these models is the data required for their application. The model proposed in this work presents the advantage of only being based on a tableting process parameter, the compression pressure, which is easily accessible since the tablets are manufactured, and by the knowledge of the tensile strength of two different compositions for a given system. 


\section{Conclusion}

In this work, the impact of operating conditions on the mechanical strength of binary tablets was evaluated, including both process and formulation parameters.

As expected, for the two diluents used in the formulation, the tablet tensile strength increased with the compression pressure. Whatever the compression pressure, the $\mathrm{CFI} / \mathrm{MCC}$ tablets exhibited a decreasing tensile strength with an increase of the caffeine content. By contrast, an opposite behavior was observed for CFI/DCPA tablets (tensile strength increasing with caffeine content). This is in accordance with the literature $[18,24]$ and attests that the choice of the ingredients is essential and could have many consequences on the quality of the final tablets.

Moreover, all of these results showed that a relationship between a process parameter and a mechanical characteristic of tablets can be found. So, a predictive model for the tensile strength of pharmaceutical tablets was developed for a given binary system as a function of the compression pressure. Constants used in the proposed model were determined from the tablet tensile strength of the two extreme compositions of each studied system. This model was validated with experimental results, for both studied binary systems and on a wide range of compositions and compression pressures which are quite commonly used in the pharmaceutical manufacturing. The proposed model is relevant for the two studied mixtures, whether it consists of two plastic compounds or a mixture containing a plastic and a fragmentary compound. Moreover, it does not require the fabrication of pure component tablets.

Finally, the tablet tensile strength can be connected directly to the compression load, which is easily accessible since the tablets are manufactured, and by the knowledge of the tensile strength of two compositions, for a chosen binary system. By extending the applicability domain of the developed model for tablets composed of three or more ingredients, it could be part of a "Quality by Design" approach.

\section{Acknowledgements}

The support of the Ministère de l'Enseignement Supérieur et de la Recherche is gratefully acknowledged. We would like to thank MEDEL'PHARM and the IVTV platform (ANR-10-EQPX-06-01).

\section{References}

[1] T. Martinello, T.M. Kaneko, M.V.R. Velasco, M.E.S.T. Taqueda, V.O. Consiglieri, Int. J. Pharm. 322, $87-95$ (2006)
[2] L.X. Liu, I. Marziano, A.C. Bentham, J.D. Litster, E.T. White, T. Howes, Powder Technol. 240, 66-73 (2013)

[3] C. Hirschberg, C.C. Sun, J. Rantanen, J. Pharm. Biomed. Anal. 128, 462-468 (2016)

[4] T. Osamura, Y. Takeuchi, R. Onodera, M. Kitamura, Y. Takahashi, K. Tahara, H. Takeuchi, Int. J. Pharm. 510(1), 195-202 (2016)

[5] M. Jivraj, L.G. Martini, C.M. Thomson, Pharm Sci Technology Today 3(2), 58-63 (2000)

[6] M.B. Tejedor, N. Nordgren, M. Schuleit, M.W. Rutland, Int. J. Pharm. 486, 315-323 (2015)

[7] A.D. Rajkumar, G.K. Reynolds, D Wilson, S. Wren, M.J. Hounslow, A.D. Salman, Eur. J. Pharm. Biopharm. 106, 88-96 (2016)

[8] C.Y. Wu, J.P.K. Seville, Powder Technol. 189, 285 294 (2009)

[9] D.A. Pai, A.A. Hayes, M.R. Okos, Powder Technol. 239, 441-450 (2013)

[10] C. Shang, I.C. Sinka, B. Jayaraman, J. Pan, Int. J. Pharm. 442, 57-64 (2013)

[11] S.M. Razavi, G. Callegari, G. Drazer, A.M. Cuitino, Int. J. Pharm. 484, 29-37 (2015)

[12] European Directorate for the Quality of Medicines and Healthcare, European Pharmacopoeia, 8th ed. (Strasbourg, 2014)

[13] F. Podczeck, Int. J. Pharm. 436, 214-232 (2012)

[14] A. Juban, C. Nouguier-Lehon, S. Briancon, T. Hoc, F. Puel, Int. J. Pharm. 490, 438-445 (2015)

[15] Y. Zhang, Y. Law, S. Chakrabarti, AAPS PharmSciTech. 4(4) 62 (2003)

[16] J. Ilkka, P. Paronen, Int. J. Pharm. 94 181-187 (1993)

[17] J.T. Fell, J.M. Newton, J. Pharm. Sci. 59 688-691 (1970)

[18] A. Michrafy, M. Michrafy, M.S. Kadiri, J.A. Dodds, Int. J. Pharm. 333, 118-126 (2007)

[19] M. Kuentz, H. Leuenberger, Eur. J. Pharm. Biopharm. 49, 151-159 (2000)

[20] N. Ramirez, L.M. Melgoza, M. Kuentz, H. Sandoval, I. Caraballo, Eur. J. Pharm. Sci. 22, 19-23 (2004)

[21] V. Busignies, B. Leclerc, P. Porion, P. Evesque, G. Couarraze, P. Tchoreloff, Eur. J. Pharm. Biopharm. 67, 507-514 (2005)

[22] C.Y. Wu, S.M. Best, A.C. Bentham, B.C. Hancock, W. Bonfield, Eur. J. Pharm. Sci. 25, 331-336 (2005)

[23] W. Duckworth, J. Am. Ceram. Soc. 3668 (1953)

[24] S. Mangal, F. Meiser, G. Tan, T. Gengenbach, D.A.V. Morton, I. Larson, Eur. J. Pharm. Biopharm. 104, 110116 (2016)

[25] A. Koynov, F. Romanski, A.M. Cuitino, Powder Technol. 236 5-11 (2013) 